\title{
The clinical relevance of laboratory prognostic scores for patients with radiosurgically treated brain metastases of non-pulmonary primary tumor
}

\author{
Anna Cho ${ }^{1} \cdot$ Helena Untersteiner ${ }^{1} \cdot$ Fabian Fitschek $^{2} \cdot$ Farjad Khalaveh $^{1} \cdot$ Philip Pruckner $^{1} \cdot$ Noemi Pavo $^{3}$. \\ Karl Rössler ${ }^{1}$. Christian Dorfer ${ }^{1}$ • Brigitte Gatterbauer ${ }^{1}$. Christoph Höller ${ }^{4}$. Manuela Schmidinger ${ }^{5}$. \\ Josa M. Frischer ${ }^{1}$ (D)
}

Received: 25 May 2021 / Accepted: 9 June 2021 / Published online: 20 June 2021

(c) The Author(s) 2021

\begin{abstract}
Purpose To investigate the clinical value of the inflammation based prognostic scores for patients with radiosurgically treated brain metastases (BM) originating from non-pulmonary primary tumor (PT).

Methods A retrospective analysis of 340 BM patients of different PT origin (melanoma, breast, gastrointestinal, or genitourinary cancer) was performed. Pre-radiosurgical laboratory prognostic scores, such as the Neutrophil-to-Lymphocyte Ratio (NLR), the Platelet-to-Lymphocyte Ratio (PLR), Lymphocyte-to-Monocyte Ratio (LMR), and the modified Glasgow Prognostic Score (mGPS), were investigated within 14 days before the first Gamma Knife radiosurgical treatment (GKRS1). Results In our study cohort, the estimated survival was significantly longer in patients with NLR $<5$ ( $p<0.001)$, LMR $>4$ $(\mathrm{p}=0.001)$ and in patients with a mGPS score of $0(\mathrm{p}<0.001)$. Furthermore, univariate and multivariate Cox regression models revealed NLR $\geq 5$, LMR $<4$ and mGPS score $\geq 1$ as independent prognostic factors for an increased risk of death even after adjusting for age, sex, KPS, extracranial metastases status, presence of neurological symptoms and treatment with immunotherapy (IT) or targeted therapy (TT).

Conclusions Summarizing previously published and present data, pre-radiosurgical mGPS and NLR groups seem to be the most effective and simple independent prognostic factors to predict clinical outcome in radiosurgically treated BM patients.
\end{abstract}

Keywords Gamma Knife Radiosurgery · Brain metastases · Prognostic scores $\cdot$ NLR · mGPS

$\begin{array}{ll}\text { Abbreviations } \\ \mathrm{BM} & \text { Brain metastases } \\ \mathrm{CI} & \text { Confidence interval } \\ \mathrm{CRP} & \text { C-reactive protein } \\ \mathrm{ECM} & \text { Extracranial metastases }\end{array}$

Josa M. Frischer

josa.frischer@meduniwien.ac.at

1 Department of Neurosurgery, Medical University of Vienna, Waehringer Guertel 18-20, 1090 Vienna, Austria

2 Department of General Surgery, Medical University of Vienna, Vienna, Austria

3 Department of Internal Medicine II, Division of Cardiology, Medical University of Vienna, Vienna, Austria

4 Department of Dermatology, Medical University of Vienna, Vienna, Austria

5 Department of Urology, Medical University of Vienna, Vienna, Austria

$\begin{array}{ll}\text { fRT } & \text { Fractionated radiotherapy } \\ \text { FU } & \text { Follow-up } \\ \text { GKRS } & \text { Gamma Knife radiosurgery } \\ \text { GKRS1 } & \text { First Gamma Knife radiosurgical treatment } \\ \text { GI } & \text { Gastrointestinal } \\ \text { GU } & \text { Genitourinary } \\ \text { GPA } & \text { Graded Prognostic Assessment } \\ \text { HR } & \text { Hazard ratio } \\ \text { IT } & \text { Immunotherapy } \\ \text { KPS } & \text { Karnofsky Performance Status Scale } \\ \text { LMR } & \text { Lymphocyte-to-Monocyte Ratio } \\ \text { mGPS } & \text { Modified Glasgow Prognostic Score } \\ \text { MRI } & \text { Magnetic resonance imaging } \\ \text { NLR } & \text { Neutrophil-to-Lymphocyte Ratio } \\ \text { NSCLC } & \text { Non-small cell lung cancer } \\ \text { PLR } & \text { Platelet-to-Lymphocyte Ratio } \\ \text { RPA } & \text { Recursive Partitioning Analysis } \\ \text { SIR } & \text { Score Index for Radiosurgery }\end{array}$


TT Targeted therapy

WBRT Whole brain radiation therapy

\section{Introduction}

Over the last decades, there has been an increase in the incidence of brain metastses (BM) due to improvements in diagnostic workups, but also in systemic oncological therapies [1]. Although lung cancer represents the most common primary tumor (PT), the incidence of BM from other PT, such as melanoma, breast, renal, colorectal cancer should not be underestimated $[1,2]$.

In BM patients, several prognostic factors, including the PT histology, patient's age, the systemic disease status, the Karnofsky Performance Status (KPS), as well as the number, size, and localization of BM, are of utmost interest [2]. Recently, several laboratory prognostic predictors for solid and metastatic cancer patients have additionally gained interest due to their simplicity and wide availability [3-5]. Therefore, leucocyte-based ratios, such as the Neutrophilto-Lymphocyte Ratio (NLR), the Platelet-to-Lymphocyte Ratio (PLR), and the Lymphocyte-to-Monocyte Ratio (LMR), have been previously reported to be prognostic for overall survival in patients with surgically treated BM [4, 6]. However, the clinical data on NLR, PLR and LMR for radiosurgically treated $\mathrm{BM}$ patients are still mainly limited to non-small cell lung cancer (NSCLC) patients [7-9].

Moreover, the modified Glasgow Prognostic Score (mGPS), consisting of albumin and C-reactive protein (CRP), has also shown to be prognostic for survival in cancer patients [10-12]. However, mGPS has rather been applied for solid cancers so far, and clinical data in radiosurgically treated BM patients are still lacking.

The aim of the study was to investigate the clinical value of these laboratory prognostic scores in radiosurgically treated BM patients of non-pulmonary PT origin.

\section{Materials and methods}

For this retrospective study, patients who have been treated between June 2012 (implementation of the new Gamma Knife ${ }^{\circledR}$ Perfexion ${ }^{\mathrm{TM}}$ at our institution) and December 2019 were included. Our inclusion criteria were patients with an age $>18$ years, at least one Gamma Knife radiosurgical (GKRS) treatment for at least one BM from melanoma, breast cancer, gastrointestinal (GI) or genitourinary (GU) cancer, and available laboratory parameters within 14 days before the first GKRS (GKRS1) [7].

After excluding patients according to our criteria, and patients lost to follow-up (FU), 340 patients could be enrolled in this study (Fig. 1).
Subsequently, all patients with available laboratory findings for the calculation of NLR, PLR, and LMR, were classified according to the previously defined cut-off-values. The cut-off values were 5 for NLR, 180 for PLR and 4 for LMR $[4,7,13]$. As previously reported, the mGPS was defined as follows: no abnormalities in CRP $(\leq 10 \mathrm{mg} / \mathrm{l})$ and albumin ( $\geq 35 \mathrm{~g} / \mathrm{l}$ ) was classified as a mGPS score of 0 , while both abnormalities were classified as a mGPS score of 2 . For patients with elevated CRP but without decreased albumin, a mGPS of 1 was allocated [14].

To review the clinical value of prognostic scores in relation to concomitant modern oncological therapies, data on immunotherapy (IT) or targeted therapy (TT) were additionally reviewed at the time of GKRS1 ( \pm 30 days). The study complied with the Declaration of Helsinki and was approved by the local ethics review committee (EK1788/2020).

\section{Radiosurgical technique}

As we have previously described, radiosurgical treatments were planned with GammaPlan and performed with Leksell Gamma Knife ${ }^{\circledR}$ Perfexion ${ }^{\mathrm{TM}}$ (Elekta AB, Stockholm, Sweden). The planning sequences were performed on a 1.5 or 3.0 Tesla magnet MRI and always included Gadolinium contrast-enhanced T1-weighted MRI sequences in axial and coronal planes [15].

The median time between initial BM diagnosis and GKRS1 was 0.5 months (0.0-99.4). About two-thirds of the study population $(204 / 340,60 \%)$ underwent only one GKRS treatment, while the others $(136 / 340,40 \%)$ received multiple treatments due to newly diagnosed BM or two-fraction dosestaged GKRS, as described before [16].

The median treatment volume was $0.6 \mathrm{~cm}^{3}(0.1-29.0)$. The prescribed doses mainly targeted the 50\% (45-90) isodose line, with a median prescription dose of $18 \mathrm{~Gy}$ (6-24) and a median central dose of 32 Gy (12-45).

\section{Outcome evaluation and statistical analyses}

According to our standard follow-up procedure, all radiosurgically treated patients were clinically and radiologically assessed in a 3-month interval. The primary study endpoint was defined as all-cause mortality. For further outcome data evaluation, a death register comparison was performed.

Categorical data were presented as counts and percentages, and continuous parameters as median and range. The chi-square, Mann-Whitney U, and Wilcoxon signed-rank tests were performed as statistically appropriate.

Median survival after the first GKRS was estimated by the Kaplan-Meier method and compared with the Log-RankTest. Univariate and multivariate Cox proportional hazard regression analyses were performed to estimate the association of the prognostic scores with mortality and to evaluate 


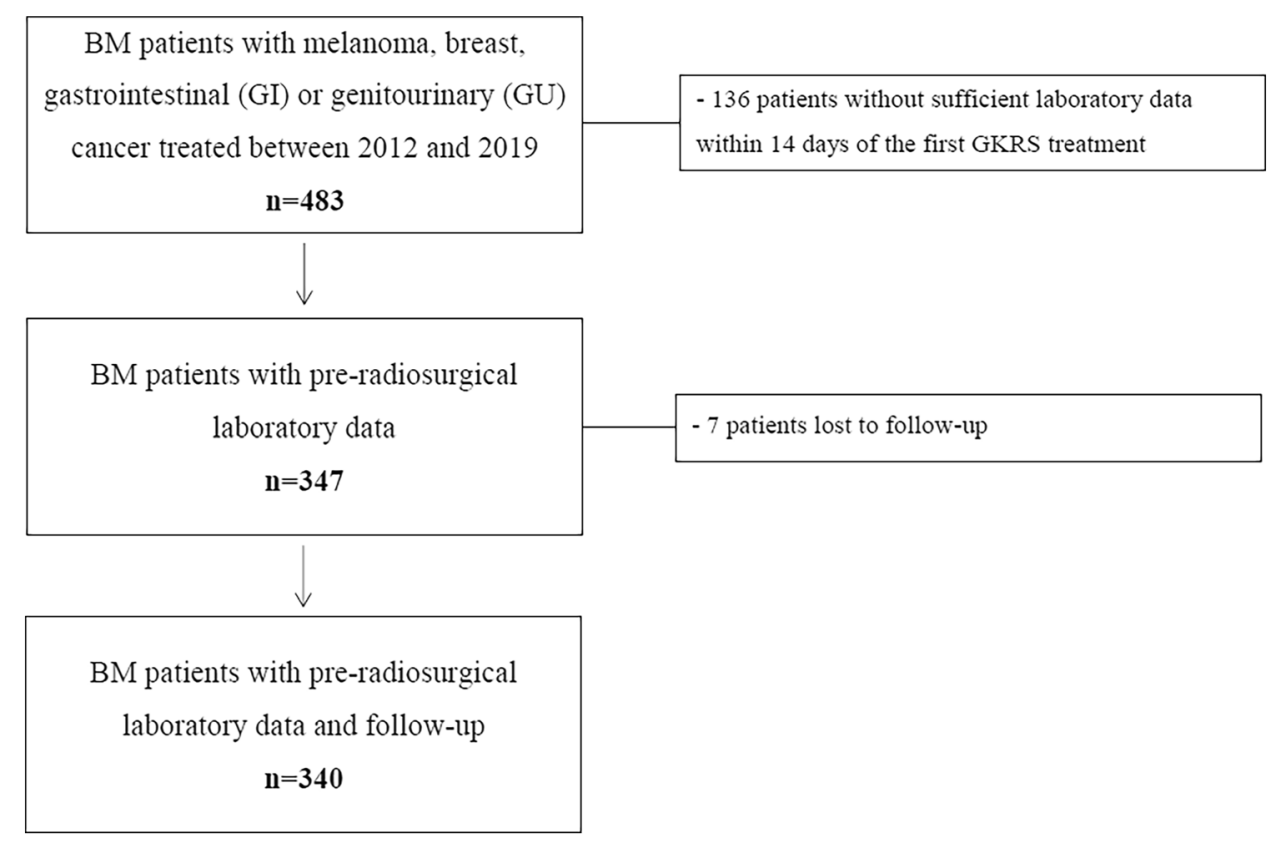

Fig. 1 Flow chart depicting the study inclusion algorithm. This figure gives an overview of our study patient selection. At our institution, 483 patients with BM from melanoma, breast cancer, GI or GU cancer were treated with GKRS for at least one BM between June 2012 (implementation of the new Gamma Knife ${ }^{\circledR}$ Perfexion $^{\mathrm{TM}}$ at our institution) and December 2019. After excluding 136/483 (28\%)

for potential confounding clinical parameters. Multivariate Cox regression analyses included sex, age group $(\leq 65$ vs. $>65 a)$, KPS group ( $<80 \%$ vs. $\geq 80 \%)$, the presence of extracranial metastases (ECM), the presence of neurological symptoms (asymptomatic versus symptomatic patients), the NLR, PLR, LMR, and mGPS groups [7].

For all tests, $\mathrm{p}$ values $<0.05$ were considered to be statistically significant. Statistical analyses were carried out with IBM SPSS Statistics for Windows (Version 26.0 Armonk, NY: IBM Corp.).

\section{Results}

\section{Patient characteristics and overall survival}

The baseline characteristics of our study population are displayed in Table 1 . The median age of the study population was 63 (29-89) years and 52\% (178/340) of the patients were female. The most common PT in our study population was melanoma $(149 / 340,44 \%)$, followed by breast cancer (94/340, 28\%), GI (55/340, 16\%), and GU (42/340, 12\%). The median time between the diagnosis of the PT and BM was 26.5 months $(0.0-354.8)$. Furthermore, the estimated median survival time was 56.0 months [95\% confidence interval $(\mathrm{CI})=46.3-65.8]$ after the initial diagnosis of the patients without archived or sufficient laboratory parameters within 14 days before GKRS1, and 7/483 (1\%) patients with lost to followup, a total of 340/483 (71\%) patients could be enrolled in this study. $B M$ brain metastases, GKRS Gamma Knife Radiosurgery, GKRS1 first Gamma Knife radiosurgical treatment, $G I$ gastrointestinal, $G U$ genitourinary

PT, 12.0 months $(95 \% \mathrm{CI}=9.6-14.3)$ after the initial diagnosis of BMs, and 9.1 months $(95 \% \mathrm{CI}=7.1-11.1)$ after GKRS1.

The estimated median survival was significantly longer in female patients $(p=0.026)$, younger patients with an age of $\leq 65$ years $(p=0.015)$, KPS of $80 \%$ or above $(p<0.001)$, and neurologically asymptomatic patients $(\mathrm{p}=0.002)$. Interestingly, the presence of ECM was not significantly associated with shorter survival $(\mathrm{p}=0.246)$.

After excluding six patients $(6 / 340,2 \%)$ without any known data on IT or TT, the survival after GKRS1 was evaluated for patients with and without IT or TT. This subanalysis showed a significantly longer estimated survival in patients with IT or TT $(182 / 340,54 \%)$ than in patients without IT or TT $(152 / 340,44 \%$; $=0.036)$.

\section{Neutrophil-to-lymphocyte ratio}

In our study cohort, NLR values could be evaluated for $311 / 340$ (92\%) radiosurgically treated BM patients. Patients with NLR $<5$ had a significantly longer estimated median survival after GKRS1 than those with NLR $\geq 5(\mathrm{p}<0.001$; Fig. 2A). For NLR groups, the univariate Cox regression model revealed a HR of $1.880(95 \% \mathrm{CI}=1.436-2.462$; $\mathrm{p}<0.001$ ). The multivariate Cox regression model, including sex, KPS groups, age groups, ECM status and the 
Table 1 Baseline characteristics of the study population

\begin{tabular}{|c|c|}
\hline & $\begin{array}{l}\text { Time of first } \\
\text { GKRS }(n=340)\end{array}$ \\
\hline Age, in years, median (range) & $60(29-89)$ \\
\hline \multicolumn{2}{|l|}{ Age groups } \\
\hline$\leq 65$ & $201(59 \%)$ \\
\hline$>65$ & $139(41 \%)$ \\
\hline Female: male ratio & 178:162 \\
\hline $\mathrm{KPS}$, in \%, median (range) & $80(40-100)$ \\
\hline \multicolumn{2}{|l|}{ KPS groups } \\
\hline$\geq 80 \%$ & $243(72 \%)$ \\
\hline$<80 \%$ & $97(28 \%)$ \\
\hline \multicolumn{2}{|l|}{ Neurological symptoms } \\
\hline Yes & $228(67 \%)$ \\
\hline No & $112(33 \%)$ \\
\hline \multicolumn{2}{|l|}{ Primary tumor } \\
\hline Melanoma & $149(44 \%)$ \\
\hline Breast & $94(28 \%)$ \\
\hline Gastrointestinal & $55(16 \%)$ \\
\hline Genitourinary & $42(12 \%)$ \\
\hline \multicolumn{2}{|l|}{ ECM Status at time of BM diagnosis } \\
\hline Yes & $302(89 \%)$ \\
\hline No & $38(11 \%)$ \\
\hline \multicolumn{2}{|l|}{ IT and/or TT } \\
\hline Yes & $182(53 \%)$ \\
\hline No & $152(45 \%)$ \\
\hline Unknown & $6(2 \%)$ \\
\hline \multicolumn{2}{|l|}{ CNS treatment before GKRS1 } \\
\hline None & $260(76 \%)$ \\
\hline WBRT and/or fRT & $23(7 \%)$ \\
\hline $\mathrm{BM}$ resection without $\mathrm{RT}$ & $34(10 \%)$ \\
\hline BM resection with WBRT and/or fRT & $23(7 \%)$ \\
\hline \multicolumn{2}{|l|}{ Localization of BM at initial diagnosis } \\
\hline Multiple & $206(61 \%)$ \\
\hline Frontal & $28(8 \%)$ \\
\hline Parietal & $25(7 \%)$ \\
\hline Temporal & $12(4 \%)$ \\
\hline Occipital & $17(5 \%)$ \\
\hline Central & $18(5 \%)$ \\
\hline Basal ganglia/brainstem/other & $8(2 \%)$ \\
\hline Cerebellar & $26(8 \%)$ \\
\hline \multicolumn{2}{|c|}{ Predicted survival after prognostic scores, in months, median (range) } \\
\hline GPA general & $3.8(2.6-11.0)$ \\
\hline GPA specific & $7.7(3.0-25.3)$ \\
\hline RPA & $4.5(2.3-7.7)$ \\
\hline SIR & $6.0(2.1-8.8)$ \\
\hline
\end{tabular}

This Table shows the detailed baseline characteristics of our 340 radiosurgically treated BM patients. Prior CNS treatment was mainly performed for distant BM. To review the clinical value of these prognostic scores in relation to IT or TT were reviewd at the time of the first GKRS treatment $( \pm 30$ days $)$

$B M$ brain metastases, $C N S$ central nervous system, ECM extracranial metastases, $f R T$ fractionated radiotherapy, GKRS Gamma Knife Radiosurgery, GPA Graded Prognostic Assessment, IQR InterQuartile Range, IT immunotherapy, KPS Karnofsky Performance Status Scale, $R P A$ Recursive Partitioning Analysis, SIR Score Index for Radiosurgery, $T T$ targeted therapy, WBRT whole brain radiation therapy presence of neurological symptoms, presented a KPS $<80 \%$ $(\mathrm{p}<0.001)$, age $>65 \mathrm{a}(\mathrm{p}<0.017)$, and NLR $\geq 5$ (HR: 1.805; $95 \% \mathrm{CI}=1.374-2.371 ; \mathrm{p}<0.001)$ as independent prognostic factors for an increased risk of death.

For the continuous NLR values, the univariate Cox regression model displayed that each increase in the NLR of 1 equaled an increase of $7.2 \%$ in risk of death (HR: 1.072; 95\% CI $=1.048-1.097$; $<<0.001)$. Moreover, the multivariate Cox regression model revealed KPS $<80 \%(\mathrm{p}<0.001)$, age $>65 \mathrm{a}(\mathrm{p}=0.024)$, but also rising NLR values (HR: $1.060 ; 95 \% \mathrm{CI}=1.036-1.085 ; \mathrm{p}<0.001)$ as independent prognostic factors for an increased risk of death.

For the outcome evaluation in relation to IT or TT, NLR was investigated separately for patients with and without IT or TT (IT or TT treatment group). Interestingly, pre-radiosurgical NLR values $<5$ were associated with a significantly longer estimated median survival in patients with IT or TT $(p=0.003)$, but also in patients without IT or TT $(p=0.002)$. Thus, in a next step, we included IT and TT groups in our multivariate Cox regression model. Even after adjusting for sex, age, KPS, ECM status, presence of neurological symptoms and IT or TT group, NLR groups remained a statistically significant independent prognostic factor for an increased risk of death (HR: $1.822 ; 95 \% \mathrm{CI}=1.384-2.399$; $\mathrm{p}<0.001)$.

\section{Platelet-to-lymphocyte ratio}

The PLR values could be evaluated for $311 / 340$ (92\%) radiosurgically treated $\mathrm{BM}$ patients. Interestingly, the estimated median survival did not show any significant differences between patients with PLR $<180$ and patients with PLR $\geq 180$ ( $p=0.226$; Fig. 2B).

\section{Lymphocyte-to-monocyte ratio}

The LMR values could be evaluated in 302/340 (89\%) radiosurgically treated BM patients. The estimated median survival was significantly longer in patients with $L M R \geq 4$ than in patients with LMR $<4$ ( $\mathrm{p}=0.001$; Fig. $2 \mathrm{C})$. Consequently, the univariate Cox regression model for LMR groups displayed a HR of 1.694 (95\% CI $=1.249-2.299$; $\mathrm{p}=0.001)$. The multivariate Cox regression model, including sex, KPS groups, age groups, ECM status and the presence of neurological symptoms, revealed KPS $<80 \%$ ( $p<0.001$ ), and LMR < 4 (HR: 1.593; 95\% CI $=1.171-2.166$; $\mathrm{p}=0.003$ ) as independent prognostic factors for an increased risk of death.

However, the univariate and multivariate Cox regression analyses did not reveal continuous LMR values as a predictor for survival.

Furthermore, pre-radiosurgical LMR values $\geq 4$ were associated with a significantly longer median survival in patients without IT or TT $(p=0.001)$. Nevertheless, this 

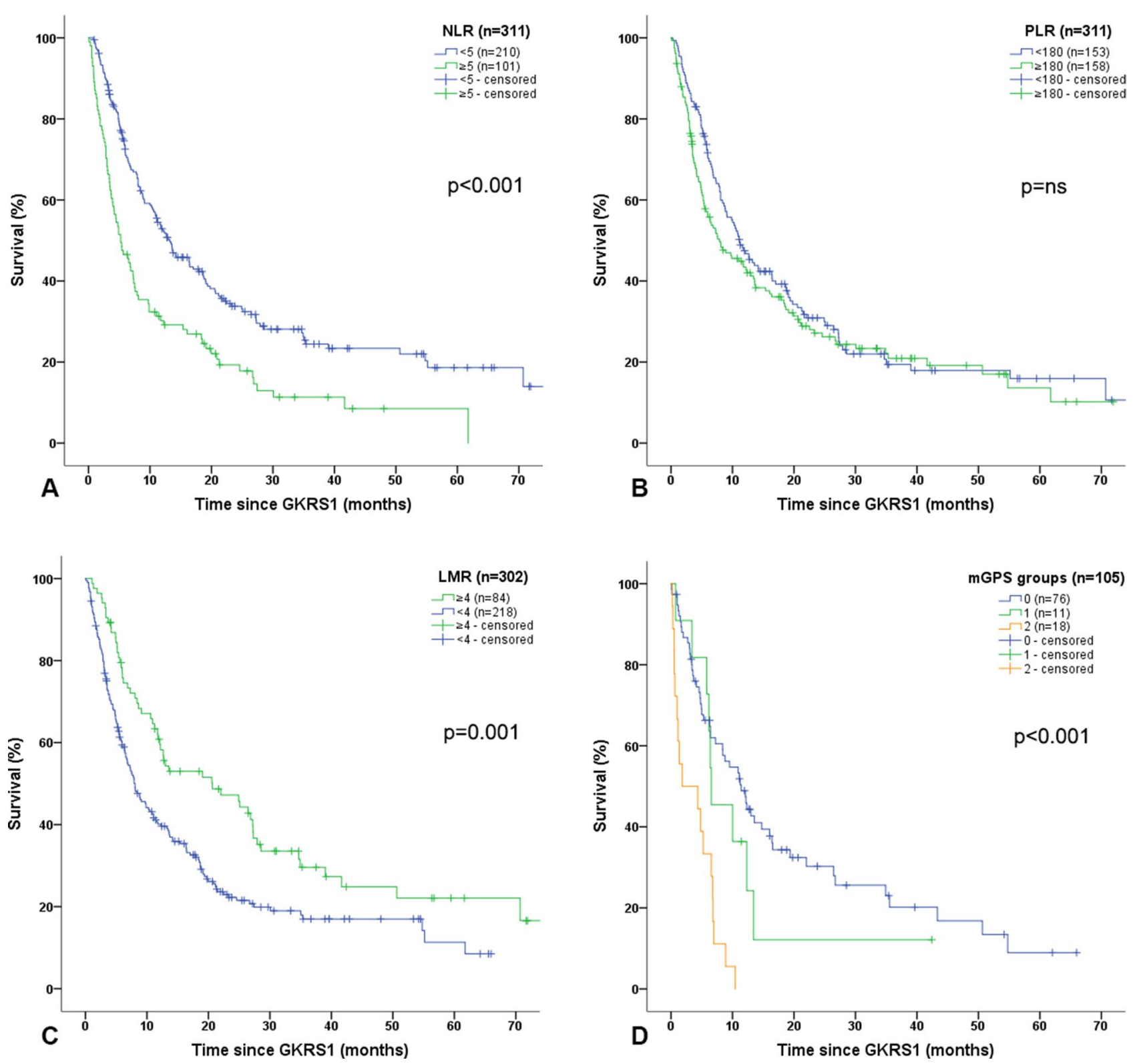

Fig. 2 Prognostic scores in relation to survival after GKRS1. A $N L R$ in relation to survival after GKRS1. In our study cohort, NLR values could be evaluated for $311 / 340(92 \%)$ radiosurgically treated BM patients. Patients with NLR $<5$ had a significant longer estimated median survival after GKRS1 (210/311, 68\%; 13.2 months, 95\% CI $=9.6-16.8)$ than those patients with NLR $\geq 5(101 / 311,32 \%$; 5.2 months, $95 \%=$ CI $3.3-7.1 ; \mathrm{p}<0.001)$. B PLR in relation to survival after GKRS1. The estimated median survival did not show any significant differences between patients with PLR $<180(153 / 311$, $49 \%$; 11.2 months, 95\% CI $=8.6-13.7)$ and patients with $\mathrm{PLR} \geq 180$ (158/311, 51\%; 7.9 months, 95\% CI $=4.2-11.6$; $\mathrm{p}=0.226$; Fig. 2B). C LMR in relation to survival after GKRS1. Due to missing monocyte values, the LMR values could be evaluated in 302/340 (89\%) radiosurgically treated $\mathrm{BM}$ patients. The estimated median survival was significantly longer in patients with $L M R \geq 4$ (84/302, 28\%;

20.6 months, $95 \%=$ CI 8.6-32.7) than in patients with $\mathrm{LMR}<4$ $(218 / 302,72 \%$; 8.0 months, 95\% CI = 6.2-9.8; $\mathrm{p}=0.001)$. D $m G P S$ in relation to survival after GKRS1. Due to missing albumin values, mGPS could only be evaluated in 105/340 (31\%) radiosurgically treated BM patients. The estimated median survival after GKRS1 was significantly longer in patients with a mGPS score of $0(76 / 105,72 \%$; 11.4 months, 95\% CI $=8.0-14.8)$ than in patients with mGPS score of $1(11 / 105,11 \% ; 6.5$ months, 95\% CI $=2.4-10.7)$ or mGPS score of $2(18 / 105,17 \% ; 1.8$ months, $95 \% \mathrm{CI}=0.0-8.1 ; \mathrm{p}<0.001) . B M$ brain metastases, $C I$ confidence interval, GKRS Gamma Knife Radiosurgery, GKRS1 first Gamma Knife radiosurgical treatment, $L M R$ Lymphocyte-to-Monocyte-Ratio, $m G P S$ modified Glasgow Prognostic Score, NLR Neutrophil-to-Lymphocyte Ratio, PLR Platelet-toLymphocyte-Ratio 
association could not be observed for patients with IT or TT $(\mathrm{p}=0.195)$. In a next step, we included IT and TT groups in our multivariate Cox regression model. Even after adjusting for sex, age, KPS, ECM status, presence of neurological symptoms and IT or TT group, LMR groups remained a statistically significant independent prognostic factor for an increased risk of death (HR: $1.571 ; 95 \% \mathrm{CI}=1.152-2.143$; $\mathrm{p}=0.004)$.

\section{Modified glasgow prognostic score}

Due to missing albumin values, mGPS could only be evaluated in 105/340 (31\%) radiosurgically treated BM patients. From these patients, the mGPS score was 0 for $76 / 105(72 \%)$ patients, 1 for $11 / 105(11 \%)$ patients and 2 for $18 / 105(17 \%)$ patients. The estimated median survival after GKRS1 was significantly longer in patients with a mGPS score of 0 than in patients with mGPS score of 1 or 2 ( $p<0.001$; Fig. 2D).

The univariate Cox regression model revealed that each increase in the mGPS score was associated with an increased risk of death (HR: $1.954 ; 95 \% \mathrm{CI}=1.460-2.613 ; \mathrm{p}<0.001$ ).

Furthermore, the multivariate Cox regression model, including sex, KPS groups, age groups, the presence of ECM and neurological symptoms, showed higher mGPS groups (HR: 1.933; 95\% CI $=1.447-2.583 ; \mathrm{p}<0.001$ ), and neurologically symptomatic BM patients (HR: $1.767 ; 95 \% \mathrm{CI}$ $=1.057-2.951 ; \mathrm{p}=0.030$ ) as independent prognostic factors for an increased risk of death.

Despite the small sample size, the correlation between shorter estimated median survival after GKRS1 and increasing mGPS could be observed for patients with IT or TT $(\mathrm{n}=55 / 105,52 \% ; \mathrm{p}<0.001)$, but also for patients without IT or TT $(n=48 / 105,46 \% ; p=0.033)$. Two of 105 patients (2\%) were excluded from this analysis due to missing data on IT or TT. Furthermore, in a next step, we included IT and TT groups in our multivariate Cox regression model. Even after adjusting for sex, age, KPS, ECM status, presence of neurological symptoms and IT or TT group, mGPS groups remained a statistically significant independent prognostic factor for an increased risk of death (HR: 1.836 ; 95\% CI $=1.361-2.476 ; \mathrm{p}<0.001)$.

\section{Discussion}

Despite the improvements in the management of cancer patients, the prognosis of patients with BM remains poor as it is still associated with short survival duration [17]. As the outcome of BM patients is known to be influenced by a variety of patient- but also tumor-relevant factors, the individual assessment for the optimal management with simple methods is crucial $[2,18]$.
In this retrospective analysis, simple laboratory parameters and their ratios, which are obtained in clinical routine, were analyzed for their prognostic values in a clearly defined cohort of Gamma Knife radiosurgically treated BM patients from different non-pulmonary PT origins (melanoma, breast cancer, GI and GU cancers).

In general, the main treatment goal for patients with BM is the local control of the metastatic lesion, in order to maintain a satisfactory quality of life while preventing death from neurological complications [17]. For local control, stereotactic radiosurgery represents a non-invasive, highly-effective method, especially for patients with multiple or deeply seated, and thereby non-resectable BM [2, 17]. Nevertheless, the therapeutic decision making for patients with multiple BM still remains challenging.

In the past, whole brain radiation therapy (WBRT) was considered to be the treatment of choice for patients with multiple BM. However, the lack of survival benefit together with the impairment in quality of life and the neuro-cognitive deterioration have recently heavily challenged the therapeutic decision for WBRT [17-19]. At our institution, stereotactic radiosurgery with a reduced prescription dose for a high number of BM is regularly performed; this procedure has also been described as an innovative therapeutic approach by other groups $[17,18]$.

Nevertheless, the decision of the therapeutic management should be made individually, depending on the clinical condition and prognosis of the patient [18]. Thus, easily available predictive scores are of a high interest in clinical routine and for clinical trial inclusion [7, 20].

Systemic inflammation was identified to be associated with cancer development, metastasis and progression. Similarly, markers of inflammation, such as CRP, albumin or leucocyte-based ratios, were shown to predict tumor progression in a variety of solid tumors $[6,8,13,14,21,22]$. So far, data on radiosurgically treated BM patients remain scarce $[5,8]$. Since NSCLC represents the most common cause of BMs, we assessed the prognostic impact of these leucocyte-based ratios in NSCLC patients with radiosurgically treated BM in a previous report [7]. The current study adds to the growing body of research on the clinical relevance of systemic inflammation-based scores, mGPS and leucocytebased ratios, in radiosurgically treated BM patients with non-pulmonary PT, including melanoma, breast, GI and GU cancers. In this study cohort, pre-radiosurgical NLR and LMR groups were generally associated with longer survival duration after GKRS1. Of note, multivariate Cox regression models, including sex, KPS, age, the presence of ECM and neurological symptoms, revealed NLR and LMR groups as independent prognostic factors for an increased risk of death of $80.5 \%$ and $59.3 \%$, respectively.

In addition to NLR and LMR groups, the prognostic values of the continuous metric values were also evaluated. 
After univariate and multivariate Cox regression models, only continuous rising NLR values were revealed as an independent predictor for an increased risk of death. Even after adjusting for age, sex, KPS and presence of neurological symptoms or ECM, each increase in the NLR of one equaled an increase of $6.0 \%$ in risk of death. A predictive value of PLR could not be observed, neither for PLR groups nor continuous PLR values.

In recent years, the advent of IT and TT has significantly changed the survival and the management of oncological patients and even of patients with multiple BM [17, 18, 23]. This benefit of IT or TT treatment was reflected in our study population as well. Patients with IT or TT showed a significantly longer survival after radiosurgical treatment than patients without IT or TT.

Thus, we additionally investigated these leucocyte-based scores for their prognostic significance in BM patients with and without systemic IT or TT at the time of first radiosurgical treatment $[15,23]$. Of note, NLR and LMR groups remained statistically significant independent prognostic factors, even after adjusting for IT or TT treatment. We have previously reported that the prognostic role of NLR is superior to PLR or LMR superior in radiosurgically treated NSCLC BM patients with concomitant IT or TT [24]. The current study confirms that NLR may represent the most important leucocyte-based ratio for radiosurgically treated BM patients. Therefore, NLR could be easily applied in clinical routine to predict clinical outcome after radiosurgical treatment, even in patients with IT or TT treatment.

In addition to the leucocyte-based ratios, the pre-radiosurgical modified Glasgow Prognostic Score represented a strong prognostic predictor for survival after GKRS1 in our radiosurgically treated BM patients. Further sub-analysis for mGPS showed that each increase in a mGPS score was highly associated with an increased risk of death, even after adjusting for sex, KPS, age, ECM status, presence of neurological symptoms and IT or TT treatment. These findings were achieved despite different PT origins and limited patient numbers in each mGPS groups.

Several potential clinical parameters, such as female sex, younger age, higher KPS, and neurological asymptomatic patients, were shown to be associated with improved survival in BM patients in the previous literature and our study population $[2,25,26]$. However, pre-radiosurgical mGPS and NLR groups remained statistically significant predictors despite these potential confounders. Thus, summarizing previously published and present data, pre-radiosurgical mGPS and NLR groups seem to be the most effective and simple independent prognostic factors to predict clinical outcome in radiosurgically treated $\mathrm{BM}$ patients.

As we and others have commented before, the ability to predict survival in cancer patients with simple and widely available prognostic scores is of clinical importance and may help to facilitate clinical decision making and appropriate stratification of future clinical trials [7, 20, 24]. Still, we would like to further comment, that, at our institution, patients in a palliative setting or with a high number of BM are regularly treated radiosurgically if any benefit from the treatment might be anticipated. Indeed, we do not want to imply that patients with unfavorable scores should not be treated. The clinical decision should always be made according to the patients' wishes and in the interdisciplinary agreement of the radiosurgeon and the oncologist. However, simple oncological scores and ratios may help the clinician to appropriately counsel their patients but should not prevent treatment a priori.

\section{Limitations}

One of our study limitations was the retrospective design in a non-randomized study cohort. Furthermore, several patients had to be excluded from the mGPS analysis due to missing pre-radiosurgical albumin values. This is explained by the fact that, so far, albumin was not considered to be clinically relevant for the radiosurgical planning MRI or treatment per se.

\section{Acknowledgements None.}

Author contributions AC: Conceptualization, Data acquisition, Methodology, Formal analysis, Data Curation, Writing- Original draft preparation. HU, FF, FK, PP, BG: Data acquisition, Writing-Review \& Editing. NP, KR, CD, CH, MS: Writing-Review \& Editing. JMF: Conceptualization, Data acquisition, Methodology, Formal analysis, Data Curation, Writing-Original draft preparation, Supervision.

Funding Open access funding provided by Medical University of Vienna. Not applicable.

Data availability Research data will not be shared.

Code availability Not applicable.

\section{Declarations}

Conflict of interest Not applicable.

Ethical approval The study was approved by the ethics review committee of the Medical University of Vienna (EK1788/2020).

Open Access This article is licensed under a Creative Commons Attribution 4.0 International License, which permits use, sharing, adaptation, distribution and reproduction in any medium or format, as long as you give appropriate credit to the original author(s) and the source, provide a link to the Creative Commons licence, and indicate if changes were made. The images or other third party material in this article are included in the article's Creative Commons licence, unless indicated otherwise in a credit line to the material. If material is not included in 
the article's Creative Commons licence and your intended use is not permitted by statutory regulation or exceeds the permitted use, you will need to obtain permission directly from the copyright holder. To view a copy of this licence, visit http://creativecommons.org/licenses/by/4.0/.

\section{References}

1. Barnholtz-Sloan JS, Sloan AE, Davis FG, Vigneau FD, Lai P, Sawaya RE (2004) Incidence proportions of brain metastases in patients diagnosed (1973 to 2001) in the metropolitan detroit cancer surveillance system. J Clin Oncol 22:2865-2872. https://doi. org/10.1200/JCO.2004.12.149

2. Soffietti R, Ruda R, Mutani R (2002) Management of brain metastases. J Neurol 249:1357-1369. https://doi.org/10.1007/ s00415-002-0870-6

3. Mandaliya H, Jones M, Oldmeadow C, Nordman II (2019) Prognostic biomarkers in stage IV non-small cell lung cancer (NSCLC): neutrophil to lymphocyte ratio (NLR), lymphocyte to monocyte ratio (LMR), platelet to lymphocyte ratio (PLR) and advanced lung cancer inflammation index (ALI). Transl Lung Cancer Res 8:886-894. https://doi.org/10.21037/tlcr.2019.11.16

4. Mitsuya K, Nakasu Y, Kurakane T, Hayashi N, Harada H, Nozaki K (2017) Elevated preoperative neutrophil-to-lymphocyte ratio as a predictor of worse survival after resection in patients with brain metastasis. J Neurosurg 127:433-437. https://doi.org/10.3171/ 2016.8.JNS16899

5. Zhang L, Hu Y, Chen W, Tian Y, Xie Y, Chen J (2020) Pre-stereotactic radiosurgery neutrophil-to-lymphocyte ratio is a predictor of the prognosis for brain metastases. J Neurooncol 147:691-700. https:// doi.org/10.1007/s11060-020-03477-w

6. Schneider M, Schafer N, Bode C, Borger V, Eichhorn L, Giordano FA, Guresir E, Heimann M, Ko YD, Lehmann F, Potthoff AL, Radbruch A, Schaub C, Schwab KS, Weller J, Vatter H, Herrlinger U, Landsberg J, Schuss P (2021) Prognostic value of preoperative inflammatory markers in melanoma patients with brain metastases. J Clin Med. https://doi.org/10.3390/jcm10040634

7. Cho A, Untersteiner H, Hirschmann D, Fitschek F, Dorfer C, Rossler K, Zochbauer-Muller S, Gatterbauer B, Hochmair MJ, Frischer JM (2021) Pre-radiosurgery leucocyte ratios and modified glasgow prognostic score predict survival in non-small cell lung cancer brain metastases patients. J Neurooncol 151:257-265. https://doi.org/10. 1007/s11060-020-03660-z

8. Chowdhary M, Switchenko JM, Press RH, Jhaveri J, Buchwald ZS, Blumenfeld PA, Marwaha G, Diaz A, Wang D, Abrams RA, Olson JJ, Shu HG, Curran WJ, Patel KR (2018) Post-treatment neutrophilto-lymphocyte ratio predicts for overall survival in brain metastases treated with stereotactic radiosurgery. J Neurooncol 139:689-697. https://doi.org/10.1007/s11060-018-2914-5

9. Shaverdian N, Wang J, Levin-Epstein R, Schaue D, Kupelian P, Lee P, Yang I, Kaprealian T (2016) Pro-inflammatory state portends poor outcomes with stereotactic radiosurgery for brain metastases. Anticancer Res 36:5333-5337. https://doi.org/10.21873/anticanres. 11106

10. Leung EY, Scott HR, McMillan DC (2012) Clinical utility of the pretreatment glasgow prognostic score in patients with advanced inoperable non-small cell lung cancer. J Thorac Oncol 7:655-662. https://doi.org/10.1097/JTO.0b013e318244ffe1

11. Lu X, Guo W, Xu W, Zhang X, Shi Z, Zheng L, Zhao W (2019) Prognostic value of the glasgow prognostic score in colorectal cancer: a meta-analysis of 9,839 patients. Cancer Manag Res 11:229249. https://doi.org/10.2147/CMAR.S185350

12. Dolan RD, Laird BJA, Horgan PG, McMillan DC (2018) The prognostic value of the systemic inflammatory response in randomised clinical trials in cancer: a systematic review. Crit Rev Oncol Hematol 132:130-137. https://doi.org/10.1016/j.critrevonc.2018.09.016

13. Guo W, Lu X, Liu Q, Zhang T, Li P, Qiao W, Deng M (2019) Prognostic value of neutrophil-to-lymphocyte ratio and platelet-to-lymphocyte ratio for breast cancer patients: an updated meta-analysis of 17079 individuals. Cancer Med 8:4135-4148. https://doi.org/10. $1002 /$ cam 4.2281

14. McMillan DC (2013) The systemic inflammation-based glasgow prognostic score: a decade of experience in patients with cancer. Cancer Treat Rev 39:534-540. https://doi.org/10.1016/j.ctrv.2012. 08.003

15. Gatterbauer B, Hirschmann D, Eberherr N, Untersteiner H, Cho A, Shaltout A, Gobl P, Fitschek F, Dorfer C, Wolfsberger S, Kasprian G, Holler C, Frischer JM (2020) Toxicity and efficacy of gamma knife radiosurgery for brain metastases in melanoma patients treated with immunotherapy or targeted therapy - a retrospective cohort study. Cancer Med 9:4026-4036. https://doi.org/10.1002/cam4.3021

16. Frischer JM, Fraller A, Mallouhi A, Vogl UM, Baier F, Ertl A, Preusser M, Knosp E, Kitz K, Gatterbauer B (2016) Evaluation of dose-staged gamma knife radiosurgical treatment method for highrisk brain metastases. World Neurosurg 94:352-359. https://doi.org/ 10.1016/j.wneu.2016.07.038

17. Hatiboglu MA, Akdur K, Sawaya R (2020) Neurosurgical management of patients with brain metastasis. Neurosurg Rev 43:483-495. https://doi.org/10.1007/s10143-018-1013-6

18. Liu Q, Tong X, Wang J (2019) Management of brain metastases: history and the present. Chin Neurosurg J 5:1. https://doi.org/10. 1186/s41016-018-0149-0

19. Chang EL, Wefel JS, Hess KR, Allen PK, Lang FF, Kornguth DG, Arbuckle RB, Swint JM, Shiu AS, Maor MH, Meyers CA (2009) Neurocognition in patients with brain metastases treated with radiosurgery or radiosurgery plus whole-brain irradiation: a randomised controlled trial. Lancet Oncol 10:1037-1044. https://doi.org/10. 1016/S1470-2045(09)70263-3

20. Sperduto PW, Yang TJ, Beal K, Pan H, Brown PD, Bangdiwala A, Shanley R, Yeh N, Gaspar LE, Braunstein S, Sneed P, Boyle J, Kirkpatrick JP, Mak KS, Shih HA, Engelman A, Roberge D, Arvold ND, Alexander B, Awad MM, Contessa J, Chiang V, Hardie J, Ma D, Lou E, Sperduto W, Mehta MP (2017) Estimating survival in patients with lung cancer and brain metastases: an update of the graded prognostic assessment for lung cancer using molecular markers (lung-molGPA). JAMA Oncol 3:827-831. https://doi.org/10. 1001/jamaoncol.2016.3834

21. Wade RG, Robinson AV, Lo MCI, Keeble C, Marples M, Dewar DJ, Moncrieff MDS, Peach H (2018) Baseline neutrophil-lymphocyte and platelet-lymphocyte ratios as biomarkers of survival in cutaneous melanoma: a multicenter cohort study. Ann Surg Oncol 25:3341-3349. https://doi.org/10.1245/s10434-018-6660-X

22. Gu L, Li H, Chen L, Ma X, Li X, Gao Y, Zhang Y, Xie Y, Zhang X (2016) Prognostic role of lymphocyte to monocyte ratio for patients with cancer: evidence from a systematic review and meta-analysis. Oncotarget 7:31926-31942. https://doi.org/10.18632/oncotarget. 7876

23. Borius PY, Regis J, Carpentier A, Kalamarides M, Valery CA, Latorzeff I (2021) Safety of radiosurgery concurrent with systemic therapy (chemotherapy, targeted therapy, and/or immunotherapy) in brain metastases: a systematic review. Cancer Metastasis Rev 40:341-354. https://doi.org/10.1007/s10555-020-09949-9

24. Cho A, Kranawetter B, Untersteiner H, Khalaveh F, Dorfer C, Rossler K, Zochbauer-Muller S, Gatterbauer B, Hochmair MJ, Frischer JM (2021) Neutrophil-to-lymphocyte ratio is superior to other leucocyte-based ratios as a prognostic predictor in radiosurgically treated non-small cell lung cancer brain metastases patients under immunotherapy or targeted therapy. World Neurosurg. https:// doi.org/10.1016/j.wneu.2021.04.033 
25. Steindl A, Yadavalli S, Gruber KA, Seiwald M, Gatterbauer B, Dieckmann K, Frischer JM, Klikovits T, Zochbauer-Muller S, Grisold A, Hoda MAR, Marosi C, Widhalm G, Preusser M, Berghoff AS (2020) Neurological symptom burden impacts survival prognosis in patients with newly diagnosed non-small cell lung cancer brain metastases. Cancer 126:4341-4352. https://doi.org/10.1002/ cncr.33085

26. Radkiewicz C, Johansson ALV, Dickman PW, Lambe M, Edgren G (2017) Sex differences in cancer risk and survival: a Swedish cohort study. Eur J Cancer 84:130-140. https://doi.org/10.1016/j.ejca.2017. 07.013

Publisher's Note Springer Nature remains neutral with regard to jurisdictional claims in published maps and institutional affiliations. 